\title{
LOS DERECHOS DE PROPIEDAD SOBRE LA TIERRA
}

\section{Reglas de juego y cambios en su institucionalidad}

\author{
Ricardo Lozano Botache \\ José M. González Afanador ${ }^{2}$ \\ Diana M. Osma $R^{3}$
}

\section{RESUMEN:}

Los derechos de propiedad sobre la tierra todavía son objeto de estudio en el siglo XXI, aunque ya debiera ser un tema superado, desde México hasta Argentina para sólo mencionar a Latinoamérica, hay cuestiones de la tierra por resolver y disculpas para asumir actitudes al respecto; aquí se presenta una revisión sobre los cambios que han sucedido últimamente en Colombia, donde la propiedad privada se inició en la época de (o con la colonización) la colonia y se ha conservado en su esencia conceptual en la etapa republicana. Se han mantenido reglas de juego tanto formales como informales, aunque recientemente se percibe el reconocimiento del derecho de uso del bien productivo tierra como el real determinante del desarrollo, por encima de la noción clásica que daba esa característica al derecho de propiedad. La reforma agraria bajo los cánones ortodoxos de la repartición de la tierra, que no sucedió, ha sido redefinida por cambios institucionales hacia el mercado de tierras, primero asistida y luego liberada al juego de la oferta y la demanda, pero entendido que se requiere un marco riguroso $\mathrm{y}$, ante todo, eficiente en asuntos como los sistemas de registro, catastro y una sólida convicción social de respeto a la propiedad privada. En este texto se tratan los cambios desde la perspectiva neoinstitucional.

\section{PALABRAS CLAVE}

Derecho de propiedad agraria, Tierras agrícolas, Mercado de tierras, Administración de la tierra.

\begin{abstract}
Property rights over land are still under consideration in the XXI century, although it should be a subject passed from Mexico to Argentina for only mentioning Latin America, there are issues of unresolved land and apologies to be assumed and attitudes towards it. Here there is a review of the changes that have recently happened in Colombia, where private property that began with the colony, has been preserved in the conceptual essence Republican period. Rules games have been kept in formal and informal fields, although it has been recently perceived recognition of the right to use the factual real productive
\end{abstract}

1 Magíster en Desarrollo Rural y en Administración de Empresas. Docente- Investigador USTAGRI Universidad Santo Tomás Bucaramanga

2 Magíster en Economía y en Administración de Empresas. Docente-Investigador Economía Social Facultad de Economia Universidad Santo Tomás Bucaramanga

3 Estudiante de Administración de Empresas Agropecuarias. Universidad Santo Tomás Bucaramanga 
land as the determinant of development, beyond the classical notion that gave the right of property ownership. Land reform under the orthodox canons of the distribution of land, which did not happen, is being redefined by institutional changes to the land market, first assisted and then released into the interplay of supply and demand, but understanding that is required rigorous framework for efficient and above all in matters such as registration systems, land and a strong social belief of respect for private property. The changes from neo institutional perspectives are managed in the text.

\section{KEY WORDS}

Land property rights, cropland, land markets, land administration

\section{Introducción}

En la discusión conceptual y en la implementación de políticas públicas en los últimos treinta años básicamente se han alternado varios enfoques de teoría económica en el mundo occidental. El neo-institucionalismo al interpretar y recientemente al proponer nuevas reglas de juego desde la perspectiva histórica y el estudio de la economía de las organizaciones sociales, ha estado presente, cada vez, con más fuerza en las decisiones públicas en América Latina desde todos los frentes de acción de los Estados y, en ello, Colombia no es la excepción. A pesar de lo dicho, no ha sido posible actualmente reconocer con detalle la incidencia del neoinstitucionalismo en el sector agrario colombiano, porque la temática como tarea de análisis es reciente y todavía abordada por los analistas en forma general y desde ópticas diferentes.

El presente escrito busca ser una contribución a la discusión sobre los mercados de tierras de uso agrícola desde el enfoque teórico neo-institucional: primero, se presenta la institucionalidad de la tierra mediante una retrospección hasta referentes antiguos donde ya empieza a notarse la necesidad de reglas de juego en esa temática y, luego, se intenta hacer discurrir el juego institucional hasta esta época, posteriormente, se presentan los derechos de propiedad sobre la tierra, entendidos desde el enfoque propuesto y, finalmente, se hace una presentación de los últimos cambios percibidos, donde los derechos de propiedad parecen evolucionar hacia los derechos de uso.

\section{La institucionalidad de la tierra}

Aunque este artículo no pretende hacer un recorrido histórico detallado, los autores consideran oportuno referir la primera institucionalización de la tierra de la que se tiene referencia esta descrita en la Biblia, el Libro de Josué, donde se narra que se enviaron tres personas por familia a recorrer la tierra y hacer un mapa y un libro 
para registrar la repartición, la orden la impartió el sucesor de Moisés en reprimenda a su pueblo por ser negligentes y no haberlo hecho antes, cuando Dios concedió la tierra a sus primeros padres. El libro Josué es el primero de los llamados históricos y desde esa comprensión, el Capítulo 18 da cuenta del más antiguo ordenamiento de la propiedad y su delimitación desde un poder coercitivo ${ }^{4}$, en este caso patriarcal sustentando en delegación divina.

Las cuestiones de la tierra tienen su institucionalidad o sus "reglas de juego" socialmente aceptadas por origen informal o consuetudinario que es el que se impone por la costumbre y por origen formal dado dentro de un marco normativo legal o de derecho positivo, y como es de esperarse, desde el enfoque neo-institucional propuesto, algunos individuos dispuestos a cambiar esas reglas de juego o por lo menos a soslayarlas buscando su conveniencia particular, porque detectan que pueden lograr apropiarse de beneficios o por inconformidad con el marco de incentivos propuestos socialmente como "óptimos" para quien respete las reglas, (De la Garza, 2005).

En Colombia el espectro de la violación de lo consuetudinario varía desde la costumbre de declarar valores inferiores en las transacciones comerciales para evadir impuestos y, a la vez, disminuir el costo de la transacción misma, un hecho para muchos "natural" en todo negocio de tierras, como lo expone Santos (2008), hasta la violación por la fuerza de los derechos de propiedad, incluida la expulsión, con la noción para el agresor, de poder utilizar con el transcurso del tiempo argumentos legales para apropiarse finalmente de ella. ${ }^{5}$ Se encuentran en la zona intermedia múltiples costumbres como el fraccionamiento en minifundios entre los herederos de una propiedad, la apropiación irregular de áreas de la nación para uso común o de fragilidad ambiental, la depredación del suelo, la incorrecta declaración del área o el valor de un predio con propósito de evasión o elusión fiscal y la acumulación de la tierra como símbolo de status social.

En la circunstancia actual de cambios incrementales en la institucionalidad de la tierra cuyos efectos son objeto de estudio y debate político, el neo-institucionalismo ayuda a lograr lo propuesto por la característica metodológica que tiene de integrar la historia con la economía y los hechos sociales, Rivas (2003, p.30) al citar las palabras de JJ. Romero expuso que:

$4 \quad$ Acerca de lo expuesto dice la Biblia, libro de Josué: ${ }^{18: 8}$ : Levantándose, pues, aquellos varones, fueron; y mandó Josué a los que iban para delinear la tierra, diciéndoles: Id, recorred la tierra y delineadla, y volved a mí, para que yo os eche suertes aquí delante de Jehová en Silo. ${ }^{18: 9}$ : Fueron, pues, aquellos varones y recorrieron la tierra, delineándola por ciudades en siete partes en un libro, y volvieron a Josué al campamento en Silo. ${ }^{18: 10}$ : Y Josué les echó suertes delante de Jehová en Silo; y allí repartió Josué la tierra a los hijos de Israel por sus porciones.

5 El Estatuto Rural Colombiano o Ley 1152 de 2007, declarado inconstitucional en abril de 2009, en el Artículo 130 refiriéndose a los derechos sobre la tierra de personas desplazadas con violencia de su propiedad, señalaba un término al afectado para retornar posesión y aprovechamiento del fundo y si así no fuere, se levantaba la medida de protección a sus derechos, quedando el bien en disponibilidad de ser ocupado por quien tuviera interés en él. 
"la cuestión institucional ha recuperado protagonismo en el análisis político y económico durante los últimos años, diversos autores han incorporado las instituciones como parte central del examen de la realidad social. El redescubrimiento de las instituciones ha abierto una agenda interesante de investigaciones en política y economía comparadas",

entonces, ya no más ruptura disciplinar entre la economía, la sociología, la historia y la ciencia política. Así mismo, María Luz Morán (1998, citada por Rivas, 2003, p. 38) ilustra sobre el marco de pensamiento aquí adoptado cuando expresa que posterior a la revolución conductista

"estamos asistiendo a un retorno importante de la categoría [institución] como variable significativa en el análisis político, que supone un intento de superar, o al menos, de completar el individualismo metodológico, tan poderoso en los años setenta y ochenta, afirmando que no existe un actor individual sino sujetos que actúan insertos dentro de complejas tramas institucionales"

La institucionalidad de la tierra ha esbozado cambios profundos pero no revolucionarios, esos cambios incrementales de los aspectos formales expresados normativamente en las reformas agrarias, en las políticas públicas de ordenamiento territorial, en la promoción de mercados de compra, arriendo y leasing de tierras y en las expresiones sociales por el acceso a su propiedad como la toma por la fuerza, la consolidación de cinturones marginales urbanos o alrededor de plantaciones agroindustriales o hasta el desplazamiento, pueden ser descritos y explicados desde este marco teórico neo-institucional, en el que se asume como cierto que la función principal de las instituciones en la sociedad es reducir la incertidumbre, al establecer una estructura estable, pero no necesariamente eficiente, de la interacción humana.

Por lo anterior puede esbozarse un dilema: ¿Las instituciones hacen rígidas las relaciones en la sociedad o son el un agente de cambio? Pues bien, la estabilidad de las instituciones de ningún modo contradice el hecho de que estén en cambio permanente, como lo dice North $(1995$, p.3)

"Partiendo de acuerdos, códigos de conducta y normas de comportamiento, pasando por leyes estatutarias, derecho escrito y contratos entre individuos, las instituciones se encuentran evolucionando y por consiguiente, están alterando constantemente las elecciones a nuestro alcance. Marginalmente los cambios pueden ser tan lentos y glaciales en cuanto a carácter, que debemos echarnos hacia atrás para percibirlos, aunque vivimos en un mundo en que la rapidez del cambio institucional es innegable".

En Colombia como regla de juego formal la propiedad privada y la propiedad pública han sido juntadas y acomodadas como una institución administrativa, en escala ascendente y según los intereses de grupos sociales o intereses políticos partidistas dominantes, desde la unidad mínima, la vereda, hasta el territorio total de la nación. 
En el campo agrario el Estado establece las reglas de juego en función de la representatividad que dentro de él tienen las clases sociales y su capacidad de operar como grupo de presión, (Machado 1991); de allí que si fuese posible proyectar en una pantalla de cine a una velocidad de un segundo por cada año la dinámica de las tierras en Colombia durante sus 190 años de vida republicana, tendríamos la representación en tres minutos diez segundos de un sistema de fronteras que crecen y decrecen, fracciones que se desprenden, áreas internas que asincrónicamente se disgregan o se integran, predios que mutan de forma y tamaño y tierras de propiedad común que cambian o se degradan; todo un sistema en permanente movimiento al ritmo de la circunstancia y el interés de la política partidista y los intereses hegemónicos que se juegan dentro del territorio. Si se superpusiera a ese juego la dinámica poblacional, ambiental, de lucha por el poder político y la dinámica económica, se tendría un complejo bastante ilustrativo pero a su vez altamente preocupante, porque los interrogantes acerca de la forma como se ha venido administrado la tierra se multiplican.

Las reglas de juego formales en cuestión de tierras han estado referidas en Colombia al reconocimiento de la propiedad pública y privada, la transferencia del derecho de propiedad y sus posibles enajenaciones; los derechos de propiedad con la noción que actualmente se tiene de ellos, surgieron luego de la independencia de España cuando se dio la promulgación por el Congreso de Villa del Rosario de la Ley de "Urgentes medidas sobre enajenación de tierras el 13 de octubre de 1821, (Banco de la República,1997) obsérvese que es un acto legislativo promulgado tan sólo tres años después de la independencia de la corona española ${ }^{6}$.

La mencionada Ley de 1821 se conservó en su esencia en el Código Civil de la República de Colombia vigente desde 1887, con adiciones y modificaciones, cuando en el libro segundo que trata "De los bienes y de su dominio, posesión, uso y goce", se reconoce y precisa el alcance que tiene el derecho de dominio o de propiedad privada, atribuyéndosele además, la característica de derecho real sobre una cosa corporal para gozar y disponer de ella (arbitrariamente) no siendo contra la ley o contra derecho ajeno. Esa capacidad, por analogía, otorgada a los dueños de la tierra, fue limitada por la normatividad ambiental, desde cuando la tierra quedó incluida en el Decreto 2811 de 1974 como parte integral del medio ambiente y, por lo mismo, patrimonio común, bajo la responsabilidad del Estado y de los particulares por ser de utilidad pública e interés social.

$6 \quad$ Esta Ley de la Gran Colombia de 1821, es uno de los sustentos jurídicos a la actual Ley de Tierras y Desarrollo Agrario de 2005 en la República Bolivariana de Venezuela, en ella se conminaba a los propietarios en 1821 a registrar sus títulos ante una oficina local de agrimensura, dentro de un plazo estipulado, y de no ser así, retornaría el derecho de dominio sobre los bienes a la República, actualmente en Venezuela si no se demuestra haber cumplido ese acto, el predio objeto intervención por la Ley de Tierras de 2005 se declara baldío de la nación. 
Acerca de la libertad en el uso y goce de la tierra como recurso natural sobre el que se puede legalmente ejercer el derecho de propiedad, desde las conferencias y acuerdos globales sobre medio ambiente realizados hacia la década de los 70, a los que Colombia adhirió, se ha estructurado el código ambiental que busca regular el dominio ${ }^{7}$ sobre la tierra en su relación con el medio ambiente y los derechos que le son inherentes, reconocido que la normatividad ambiental puede tener mayor fuerza coercitiva que algunas normas del derecho civil, al provenir de acuerdos de carácter internacional.

La propiedad de la tierra definida y delimitada como pública y privada, en las constituciones políticas colombianas en especial la de 1991, han hecho énfasis respecto de la propiedad privada en que:

"es una función social que implica obligaciones y como tal, le es inherente una función ecológica. El Estado protegerá y promoverá las formas asociativas y solidarias de propiedad (...)" (Constitución Política de Colombia, 1991).

Esta cita constitucional y la cumbre mundial sobre medio ambiente celebrada en Río de Janeiro en 1992 y a la que Colombia adhirió, sirvieron como fuente de inspiración para la promulgación de Ley 99 de 1993 que creó el Ministerio del Medio Ambiente y dio origen a las diferentes Corporaciones Autónomas Regionales. La adopción de la nueva institucionalidad y de los instrumentos de política ambiental en los países adherentes hizo que los derechos sobre la tierra desde los años 90 del siglo pasado, quedaran también dentro de la esfera de lo ambiental.

Con el acentuado interés político de disminuir la presión social por el acceso a la propiedad de la tierra, las leyes 200 de 1936, 135 de 1961, 30 de 1988, y 160 de 1994 pretendieron redistribuirla desde la premisa de necesaria recomposición de la estructura agraria por su inequitativa condición bimodal, proponiéndose desde su articulado erradicar el latifundio y el minifundio, con procedimientos para aclarar los derechos de propiedad, distribuir las tierras baldías de la nación con vocación agrícola y redistribuir la tierra privada ya laborable pero ineficientemente explotada, persiguiendo el fin superior de la "equidad social para la población campesina e indígena".

La Ley 160 de 1994 realizó los primeros intentos de aproximación a formas nuevas de acceso al uso de la tierra a través del mercado de ese bien, promoviéndolo como alternativa a la reforma de la estructura agraria, lo fundamental de esta Ley es que en ella está claramente incluida la función "productiva agrícola" que tiene el bien Tierra, afirmando su rol de bien productivo fundamentalmente capaz de generar ingresos y, por esa vía, riqueza. En este aspecto es pertinente articular el intento de promover el mercado de tierras con el cambio institucional y acudir a la reflexión

7 El derecho de propiedad suele ser invocado en textos jurídicos como derecho de dominio que viene de dominus, que quiere decir: señor, amo e implica la potestad del dueño sobre una cosa corporal. 
que hace Valdivieso (2001) sobre Demsetz, Coase y Williamson acerca de los derechos de propiedad y costos de transacción, en el sentido que ellos consideraron, que los individuos se sienten motivados a empeñarse en actividades de producción e intercambio si existe una estructura de incentivos y un definido marco de derechos de propiedad, con mecanismos que los aseguren. Comprendiéndose que establecer y mantener los derechos de propiedad implica costos de transacción, como la administración, regulación y la medición, en el caso muy aplicado de las tierras que, a su vez, requieren una institucionalidad con poder para preservar los derechos y con funcionamiento eficiente para minimizar los costo que le son inherentes.

La preservación de los derechos se ha apoyado en Colombia con el servicio de catastro, el registro de la tradición inmueble y la titulación de tierras baldías.

El sistema catastral es el conjunto de subsistemas que permiten la obtención de datos e información tanto espacial como descriptiva para la evaluación inmobiliaria catastral de naturaleza urbana o rural de un determinado territorio. (Organización de las Naciones Unidas para la Alimentación y la Agricultura [FAO], 2003). Al ampliar el concepto, el inventario de tierras o catastro tiene tres funciones básicas: la fiscal que gestiona para el Estado y para los particulares las tarifas impositivas por ejercer el derecho de propiedad u ocupación, la jurídica que aporta los elementos básicos para clasificar o identificar la propiedad pública o privada, y la física que informa sobre el inventario de tierras desde su condición material de topografía y área. El Decreto Ley 1250 de 1970 expresa:

"Artículo 72.- El catastro estará constituido por un conjunto de documentos de los cuales se obtenga una relación de los elementos de la propiedad inmueble del país, su descripción física, su valor económico y su situación jurídica."

El servicio de Registro de Instrumentos Públicos y Privados es el encargado de certificar en nombre del Estado Colombiano

"todo acto, contrato, providencia judicial, administrativa o arbitral que implique constitución, declaración, aclaración, adjudicación, modificación, limitación, gravamen, medida cautelar, traslación o extinción del dominio u otro derecho real principal o accesorio sobre bienes raíces, salvo la cesión del crédito hipotecario o prendario" (Decreto Ley 1250 de 1970).

El servicio de titulación de tierras es el instrumento de gestión a través del cual se formaliza el traslado del dominio de la tierra por parte de la nación, a quien la haya ocupado por no menos de cinco años o a una entidad de derecho privado que se proponga adelantar proyectos agropecuarios. Se comprenden algunos requisitos previos de uso y la condición fundamental de ser tierra de la Nación. La titulación de tierras ha sido un instrumento para la administración de las tierras baldías y a la vez un programa de reforma agraria que tiene por objeto la asignación de tierras a desposeídos de ellas u ocupantes con derechos precarios. 
La Ley 160 de 1994 define claramente la titulación de tierras como un proceso agrario. Otra forma agraria de administrar tierras ha sido la clarificación de la propiedad y las parcelaciones. Estos instrumentos han complementado la política agraria tratando de resolver inconsistencias en los derechos de propiedad o redistribuyendo tierras, antes de propiedad privada, como alternativa a la demanda de tierras hecha por la población campesina.

Los instrumentos de gestión antes mencionados no han resultado eficientes, tanto así que el mismo Instituto Geográfico Agustín Codazzi reconoció en el documento técnico de enero del 2004 que sustentó su reestructuración, la necesidad de crear un Sistema Nacional de Información Oficial en Colombia, pues: "se necesita resolver las duplicidades de producción de información y competencias entre el DANE, IDEAM, INGEOMINAS, IGAC e INCORA (hoy parte del INCODER)" la situación se concreta al verificar cómo se expiden en Colombia planos prediales que soportan actos administrativos de adjudicación de tierras producidos por el INCODER, que requieren otro acto oficial de reconocimiento y aprobación en el Instituto Geográfico Agustín Codazzi IGAC para ser ingresados al catastro.

Martínez y Ubaque (2002) y Barnes (2002) coinciden en sus estudios sobre el catastro colombiano y latinoamericano, en la necesidad de su actualización y consideran importante actuar en tres aspectos fundamentales: La actualización de las metodologías y tecnologías utilizadas en el proceso catastral, la producción de información eficiente de acuerdo a las necesidades de los usuarios y el aseguramiento de rápido acceso a la información.

Desde otro punto de vista, las oficinas de Registro de Instrumentos Públicos operan y expiden certificados de tradición de predios rurales que no tienen número predial, craso error de identificación y origen de inseguridad en los derechos de propiedad, y las notarías aún permiten el uso de formas arcaicas, por imprecisas, en la definición e identificación de linderos prediales. La inseguridad es tal, que un certificado catastral por ejemplo, cita antes de la firma del funcionario que lo expide, que no puede dar fe de nada distinto a la mera inscripción en el inventario de tierras de alguien que ocupaba un inmueble al momento de la visita de formación o actualización catastral; no hay responsabilidad más allá de la transmisión de un dato tomado en campo por un contratista encargado de llenar un formulario.

Otra cuestión por ver es cómo los playones comunales, las sabanas naturales, los esteros, las zonas de aguas máximas de las corrientes fluviales, las playas marinas y todas las tierras de fragilidad ambiental no han sido delimitados técnicamente, de forma que se proteja la propiedad pública o común; en la normatividad ambiental y civil se les cita como bienes comunes e imprescriptibles pero no existe un inventario y menos aún una demarcación que les individualice para establecer el límite entre 
lo público y lo privado; no hay cartografía oficial georreferenciada ${ }^{8}$ y materializada sobre esos bienes de uso común por lo que físicamente son indeterminados y es ineficiente su administración; de hecho, las estadísticas del censo de cultivos ilícitos en Colombia disponible en la página Web de la Dirección Nacional de Estupefacientes [DNE] (2008), refleja en su contenido que se está lejos de una administración correcta de las tierras no aptas para la agricultura, pero de interés ambiental, y que están conexos otros problemas como el uso y ocupación ilegal y la presencia de actores armados dueños de los cultivos de especies para la producción de sustancias psicoactivas de comercialización ilegal.

Ante el problema de carencia de información de tierras totalmente veraz, pertinente y oportuna para hacer valer derechos de propiedad, que se configure como un sistema que sustente con solvencia la institucionalidad de la tierra, aparecen inequidades que dejan a favor del mejor informado la posibilidad de acceder al recurso con ventaja sobre el que desconoce información, este último paga más caro, toma malas decisiones de compra e incluso pierde incentivos o derechos, sean ellos subsidios o herencias, por ejemplo. Kalmanovitz (2003), explica que el neo-institucionalismo critica la ortodoxia económica introduciendo una psicología experimental para sustituir el utilitarismo y su proceso de optimización, lo que propone al individuo como un agente menos racional y soberano que el supuesto por la teoría neoclásica. Se establece así, según el autor, el ejercicio de una racionalidad limitada por las carencias de información y por los procesos cognitivos de los agentes y lo común es que los agentes dispongan de información incompleta, que sea costoso conseguir información adicional y que incluso, con buena información, los modelos mentales de los agentes que los interpretan operen de manera equivocada frente a la realidad. Por lo que sería posible entonces, que no se puedan tomar buenas decisiones cuando las instituciones están montadas sobre sistemas políticos que favorecen a muy pocos, o que no tienen manera de orientar de manera cooperativa los intereses sociales; lo que sucedería entonces, sería la captura de rentas y el incentivo a la redistribución de la riqueza y no la generación de la misma.

Bejarano (1998) expresa que en cuestión de tierras agrarias hay negocios en los que uno de los agentes tiene una información de la que carece el otro, lo que conlleva a que el no informado salga perjudicado, esto se entiende como información asimétrica. En un medio institucional regulado por un mal gobierno los accesos a la tierra proveen una renta que es compartida entre cazadores de renta y terratenientes y a veces con el funcionario que rubrica el privilegio, esta puede ser una explicación al fracaso de los modelos de reforma agraria marginal distributiva que se intentó en algunos países latinoamericanos. La información es costosa y el actor que busca

8 La georreferenciación es la determinación de un punto geográfico respecto de un sistema de coordenadas aceptadas como oficiales en un determinado país y reconocidas por los Estados en función del límite territorial y del reconocimiento del límite de la propiedad privada. Actualmente se opera mediante sistemas satelitales de posicionamiento. 
ese activo nunca sabe cuándo ha recopilado suficiente de ella para tomar buenas decisiones. En algunos sitios de Colombia como en el Departamento de Santander, Santos (2008) encontró en su trabajo de investigación sobre los factores que inciden en la formación del precio de la tierra, que la seguridad o la paz se incluyen en el precio final, este es un dato no expreso en una negociación, no obstante, transar el bien también implica un compromiso y aceptación de la institucionalidad o "autoridad" de ese sitio.

\section{Los derechos de propiedad}

Los derechos de propiedad sobre la tierra son entendidos, de forma general, en Colombia así: desde el marco constitucional los derechos referidos al límite territorial, al derecho a la propiedad y a la función social de la propiedad; desde el marco de política pública referidos a la distribución y redistribución mediante la adopción de programas de reforma agraria establecidos por Leyes y Decretos, y por fuera del marco regulador agrario, como un bien objeto de transacciones, con costumbres generalmente aceptadas en la forma de intercambiar, como hacer permutas de tierra por otros bienes, con costumbres comerciales específicas, como el pago a plazos cortos y ante un notario, y con unas normas de catastro y registro que son referentes como defensa del interés general y de los derechos de propiedad, a partir de la publicidad de los actos de transferencia del dominio y de la limitación que sobre él se impongan o se acepten, hipotecas o embargos.

Desde el enfoque que se está presentando, Demsetz (1967) expone que los derechos de propiedad son un instrumento creado por la sociedad y que su importancia se fundamenta en el hecho de ayudar a formar las expectativas que pueden sustentarse razonablemente en las relaciones con otros, expresa el autor citado que las expectativas encuentran su manifestación en leyes, hábitos y costumbres de una sociedad, por lo que el propietario de derechos de propiedad posee el consentimiento de sus pares para permitirle actuar de determinadas maneras, y por eso mismo, un propietario espera que la sociedad a través de sus instituciones impida que otros interfieran en sus propias acciones a partir de que tales acciones no están prohibidas en la especificidad de sus derechos.

Usaquen (2008) se acoge al postulado sobre los derechos de propiedad de la nueva economía institucional y señala que los derechos de propiedad subyacen a las posibilidades del intercambio y ya no se consideran un elemento exógeno, como sí lo son para los neoclásicos desde su supuesto de un intercambio libre y voluntario entre los agentes del mercado; aquí en cambio, desde el abordaje institucional se convierten en el mecanismo que coordina las diferentes relaciones entre agentes económicos condicionantes de la calidad de vida, presentándose los derechos como una institución estratégica que por su grado de impacto no puede ser ignorada. 
El reconocimiento de los derechos de propiedad asegura al dueño la opción de beneficiarse por el ejercicio del derecho potestativo de dominio sobre algo, pero no exime de la posibilidad de generarse a sí mismo, al dueño, un efecto perverso no esperado o efectos perversos o benéficos por el ejercicio del derecho, comúnmente llamados externalidades a otros. Las externalidades suelen incidir principalmente sobre terceros no involucrados en un intercambio de derechos. Esencialmente desde la exposición de Demsetz (Op. cit.), una externalidad se manifiesta cuando una de las personas que interviene en un intercambio considera muy alto para sus intereses el costo de la decisión que debería tomar para evitarla.

Entonces, una función primaria de los derechos de propiedad es la de promover incentivos para alcanzar una mayor internalización de las externalidades y se comprende así que el costo de una transacción de derechos entre partes o la internalización de efectos externos, probablemente perversos, debe razonablemente exceder la ganancia de su internalización, o expresado en otra forma: los derechos de propiedad se desarrollan o institucionalizan para internalizar externalidades cuándo las ganancias de esa decisión superan sus costos. Vogelgesang (2003) afirma que los derechos de propiedad ganan interés social cuando incentivan el uso eficiente de la tierra y las inversiones, reducen las asimetrías de la información y facilitan las transacciones con mercados financieros.

Ante lo expuesto, los derechos de propiedad se encuentran en una doble comprensión, o son una institución económica o son una institución del derecho, o tal vez, la frontera entre lo que es lícito intercambiar y la obligación de cumplir en el intercambio se hace difusa al punto que como lo expresara Bejarano (1999), "el derecho de propiedad es el instrumento de constitución del mercado, el contrato, su mecanismo de facilitación y en la responsabilidad civil el sustituto que garantiza su cumplimiento" por lo que para Bejarano el sistema jurídico puede llegar a ser un metamercado institucional, con la función precisa de ser un instrumento de asignación y distribución de recursos de acuerdo con las exigencias de la eficiencia económica y por ello, las sanciones son como los precios por eso las personas o entes económicos responden a las sanciones como se responde a los precios, consumiendo menos del bien más caro. Ante la actividad más sancionada se está menos propenso a incurrir en su violación. En relación con los derechos de propiedad sobre la tierra, desde esta perspectiva, una dura sanción o un alto impuesto, pueden ser la partida para respectivamente evitar el desplazamiento forzado, la elusión fiscal o el acaparamiento especulativo o la acumulación latifundista.

La tierra es un bien clave para algunos de los sectores pobres del medio rural y todavía puede ser la base para el desarrollo económico y social. Deininger (2003) expone que es muy importante que los países usen el recurso tierra de la manera más efectiva, centrándose en las instituciones que dan forma a la evolución de los derechos sobre la tierra, en la prevención de posibles fuentes de inseguridad sobre 
la tenencia y en las formas en que la acción de la comunidad o del gobierno pueden ayudar a reducir esa inseguridad y a crear una base para un uso más efectivo de la tierra, para promover así el crecimiento y también la reducción de la pobreza.

Los derechos de propiedad sobre la tierra para Deininger, son normas sociales que regulan la distribución de los beneficios acumulados gracias a los usos específicos dados a una cierta parcela de tierra. Desde su perspectiva varios argumentos respaldan que la estipulación de tales derechos sea de carácter público: a) el alto costo fijo de la infraestructura institucional necesaria para establecer y mantener los derechos de propiedad sobre la tierra obliga a que ella así lo sea o, por lo menos, la reglamentación pública de los mismos; b) los beneficios derivados de la posibilidad de transferir los derechos de propiedad sobre la tierra sólo se consiguen cuando tales derechos están reglamentados por leyes y pueden ser fácil e independientemente verificados; y, c) porque de no estar lo derechos amparados por un poder de gobierno dispuesto a hacerlos respetar, los propietarios y los empresarios estarán forzados a gastar recursos para defender sus reclamos de propiedad, por ejemplo, mediante vigilancia particular, muros, cercas o vallados, que no sólo son ruinosos socialmente, sino también representan una desventaja desproporcionada para los pobres, que cuentan con menos posibilidades de enfrentar tales gastos.

Confluye la literatura de las cuestiones agrarias que un motivo que llevó a la evolución de los derechos de propiedad fue la respuesta a mayores retornos sobre la inversión en usos más intensivos de la tierra debidos al crecimiento de la población o a oportunidades que surgieron de una mayor integración del mercado y de los avances técnicos. A la vez, los derechos de propiedad sobre la tierra no son muy importantes cuando hay abundancia de tierras. En el transcurso del desarrollo, la necesidad de sostener una gran población exigirá inversiones en la tierra que los productores estarán más dispuestos a realizar si sus derechos de propiedad están asegurados (Boserup, 1965, citado por Deininger, 2003). Las innovaciones institucionales apropiadas pueden generar un círculo virtuoso de más población y más inversión en la tierra, crecimiento económico y mayor bienestar (Hayami y Ruttan, 1985), al mismo tiempo que si las instituciones no administran los derechos de propiedad sobre la tierra en respuesta a estas demandas, se puede generar un conflicto y socavar el potencial productivo y económico de las sociedades.

\section{Cambios en la institucionalidad de la tierra}

Hasta tres o cuatro décadas atrás era común referirse a las cuestiones rurales como sinónimo de las cuestiones agrícolas, actualmente es más claro que lo rural es lo que está en relación con los recursos naturales, por lo que sistemas extractivos como la minería, la pesca y los aprovechamientos forestales; además, sistemas de producción de servicios como el ecoturismo, la educación, el comercio, la salud, los sumideros de desechos y hasta la industria rural, son definitivamente subsistemas del 
medio rural y en ese sentido, uno de los subsistemas presentes en el medio rural es el sistema de la agricultura, un subsistema productivo que opera con reglas propias que le hacen capaz de generar bienes agrícolas o pecuarios de uso alimentario o no alimentario, y en el que esas reglas de juego se reconocen como la institucionalidad agraria, lo agrario o la cuestión agraria.

La tierra rural es el sustrato sobre el que se dan hechos sociales en íntima relación con la cuestión agraria y la disponibilidad de tierra es una aspiración de los habitantes rurales, aunque no necesariamente para uso agrícola, se busca acceder a ella con propósitos de desarrollo familiar o empresarial y en ese sentido los gobiernos coinciden en la búsqueda de instrumentos de políticas que faciliten el acceso a la tierra por el potencial de aumentar la producción agrícola y el crecimiento del ingreso agregado, que ayudan a reducir la pobreza y la desigualdad, la mejora del medio ambiente a través del uso adecuado y proporcionan la base para un gobierno efectivo capaz de procurar la convivencia (Janvry y Sadoulet, 2005) .

Por lo ya dicho, no se puede continuar este articulo sin tocar algo que le es inherente: el cambio en la interpretación de la categoría social "campesino" porque es él el sujeto del cambio institucional, finalmente la tierra es y será la misma como elemento físico, se acude aquí a Pérez y LLambí (2007) y su argumento desde un punto de vista antropológico, cuando exponen que la identidad campesina es un término del imaginario colectivo que permite la articulación entre los intereses individuales y los intereses comunes de una población, según la perspectiva normativa o cognitiva de los propios actores. Explican Pérez y Llambí, que el elemento integrador puede ser: un territorio común, la etnicidad, la ocupación, la religión, la clase social ó el género. Así, dependiendo de cuáles son los principales puntos de quiebre en la estructura social de una sociedad, continúan los autores, estas diferencias generarán o no identidades colectivas y alianzas entre diferentes estratos y categorías sociales. Campesino y empresario rural capitalista son categorías ahora amplias, según los autores de referencia en éste párrafo, de modo que es probable encontrar familias campesinas productoras con orientación abiertamente definida al mercado o también en casos extremos, una explotación agrícola extensiva sin criterio mercantil, algo que no se concibió posible dentro la literatura clásica de la economía del siglo pasado.

Lo agrario es también, entre todo lo expuesto, la negociación de la agricultura con el resto de la sociedad, históricamente lo agrario es la relación de poder que ha sido capaz de influir en la elección de presidentes y nombramiento de ministros en Colombia cuando se reconocía la base de la economía como agraria, pero no resolver los problemas agrarios de inequidad tuvo como efecto una baja transición hacia la industrialización y los cinturones de miseria urbanos. La política pública privilegió lo agropecuario sobre lo agrario durante las últimas décadas del siglo pasado, cuando se necesitaba afianzar la capacidad de retención de población en el medio rural y paralelamente desarrollar en el sentido amplio del concepto de la agricultura. 
Los usos diversos que actualmente se dan al recurso natural como las actividades mineras, eco-turísticas, turísticas, artesanales, industriales, agroindustriales y de agricultura alternativa, al igual que el debate mundial sobre el medio ambiente y, hasta ocasionalmente, discusiones sobre la conformación de regiones y el ordenamiento territorial, parecen haber generado una dinámica que deja fuera de contexto la política pública que tenía como axioma la vocación agrícola de la población rural, la superación de la pobreza de las familias rurales mediante la asignación de tierras por ellos mismos colonizadas o a través de colonizaciones dirigidas por el propio Estado o por el fraccionamiento de unidades productivas para ser entregadas mediante programas de reforma agraria a familias pobres en forma de parcelaciones. El ingreso rural por actividades agrícolas ha disminuido, al punto que en los años noventa fue cercano a tan sólo el 11\% del PIB y, en contraste, el ingreso no agrícola es el 53\% del ingreso rural, este cambio señala el crecimiento de otras actividades rurales y claramente reglas de juego muy diferentes, (Departamento Administrativo Nacional de Estadística [DANE], 2009).

Actualmente es reconocida la necesidad de ir más allá de la reforma agraria distributiva de tierras de uso agrícola, trascender la tenencia de la tierra como objetivo. En la literatura de la cuestión agraria se encuentra que el conocimiento y la capacidad de crear valor son los reales determinantes del desarrollo de la población rural, Balcázar, et al. (2003) al igual que Echeverría (1998) explican que junto al conocimiento también debe estar la posibilidad de acceso a los recursos, mas no necesariamente a la propiedad de los mismos, por lo que resulta urgente resolver, es la movilidad o facilidad con que se accede a los recursos sobre los que se aplica el conocimiento, para ellos, y los autores de este artículo lo reconocen como muy válido, los determinantes fundamentales de la capacidad de creación y apropiación de riqueza, de poder político y de generación de valor ya no están relacionados con la distribución de la propiedad de los factores productivos, sino que ahora lo están con la capacidad y fertilidad del conocimiento y de la mente de las personas que los utilizan y gestionan para crear valor, prosperidad y reconocimiento político y social. De forma tal que las instituciones o las reglas de juego deben evolucionar hacia la promoción y aseguramiento de posibilidades de acceso a derechos de uso como los arriendos, el leasing y la compraventa de tierras en mercados cada vez más abiertos y transparentes en los que el Estado poco a poco pierde su protagonismo inicial de promotor que subsidia operaciones del mercado de tierras.

En todo caso, la regularización de los derechos de propiedad es un requisito para el funcionamiento efectivo de los mercados de tierra, porque ellos son el marco institucional que da garantías a quienes intervienen en la compra o sesión temporal o permanente de los derechos de propiedad o de uso. En ese sentido, dice Molina 
(2009) que los programas de titulación de tierras pueden contribuir directamente a un mejor funcionamiento de los mercados, ello se entiende por la visibilidad que toma la propiedad cuando se pasa de mero ocupante a titular de derechos, se entiende complementario un sistema de catastro y registro de la propiedad que sean ágiles y baratos de modo que pequeños propietarios de escasos recursos puedan fácilmente registrar sus transacciones (Williamson, 2008). De otra manera, este segmento de propietarios y propiedades difícilmente podrán participar eficientemente y por tanto, beneficiarse de los mercados de tierra.

Se recuerda en estas líneas la necesidad de un sistema de identificación predial idóneo, que supere la descripción colonial de la propiedad basado en referencias arcifinias (límites naturales) o en hitos removibles, y pasar prontamente a un sistema de coordenadas georreferenciadas por sistemas geométricos de control satelital y reconocidas oficialmente para la definición de límites privados, administrativos e internacionales, este requerimiento en si mismo cambia radicalmente las reglas de juego y la costumbre de eliminar cercos o vallados con la pretensión de cambiar linderos, pues el sistema georreferenciado, como herramienta tecnológica es independiente de cualquier hecho material sobre cosas físicas puestas sobre la tierra, (Ting \& Williamson, 2009).

Por otra parte, el acceso a la tierra por medio del mercado se reconoce que necesita un impulso de un financiamiento inicial blando, capital para acceder a la tierra y apoyo complementario a la producción agrícola para facilitar la inserción exitosa de los beneficiarios al mercado. Este tipo de programas de acceso a la tierra es facilitado cuando en el proceso de transferencia participan de forma activa los beneficiarios de los mismos. Es decir, participar en la selección de las tierras, en las transacciones de compra venta y en el diseño de planes de apoyo complementario a la producción.

Los programas de titulación de tierras no necesariamente tienen un efecto inmediato, natural y de forma similar en los diversos grupos de beneficiarios, ya que en ello influye la heterogeneidad de los mismos y el contexto macroeconómico en el cual esos programas tienen lugar. Al respecto, es necesario formular políticas que reconozcan y respondan a las diferencias entre los distintos tipos de agricultores y a las condiciones impuestas por el entorno en el que operan. Afirma Rojas (2006) que los trabajos de investigadores y profesionales del desarrollo han reconocido desde hace tiempo que proporcionar a los pobres acceso a la tierra y primordialmente mejorar su capacidad para hacer uso efectivo de la que ocupan es esencial para reducir la pobreza y para dar poder de decisión a los pobres y a las comunidades. Hace especial énfasis el autor en la importancia que tiene la tierra para la mujer rural, aunque ha estado en desventaja para acceder a ella, reconociendo que es decisivo ese activo en la estabilidad económica y sico-social de una familia. 


\section{Consideraciones finales}

A diferencia de las décadas anteriores cuando las reformas agrarias inducidas por el Estado eran el principal instrumento de política para abordar el problema de la pobreza y marginalidad rural, las iniciativas de política pública en América Latina acuden a la promoción del mercado y la optimización de sus instrumentos, esta tendencia exige, a su vez, la búsqueda de transparencia por lo que se acude a lo que ha expresado Molina (op. Cit.) en el mismo sentido: que al ser el mercado el principal distribuidor de recursos en el sector rural, tanto de tierra como de capital de trabajo se requieren ciertas condiciones relacionadas a los factores de producción como: a) derechos de propiedad claramente definidos y legalmente reconocidos para lo cual es necesario la legislación correspondiente; b) un sistema de catastro y registro de la propiedad que sea ágil, efectivo y confiable y mecanismos de resolución de conflictos sobre derechos de propiedad; $y, c)$ sistemas de información, sobre los factores de producción, de modo que los diversos agentes en el mercado puedan participar y operar en el mismo de forma eficiente y efectiva.

Se reconoce también que los recursos informáticos sobre la tierra obtenidos con métodos topográficos y tecnologías espaciales actuales específicamente llamados geomáticos son decisivos en la administración de la tierra para el desarrollo, como lo aseguran Mckenna y Urban (2008) y siguen requiriendo, como es evidente, los mismos datos que al comienzo de este artículo fueron pedidos en la cita del libro de Josué: que se levanten los datos en el campo y se consiga una correspondencia razonable y por lo mismo respetable socialmente, entre la realidad física y la realidad jurídica, acto que en ese tiempo también se llamó el libro de registro.

Reconocido el ritmo decreciente del porcentaje de participación del PIB agrícola en los países en desarrollo, también es necesario no perder la percepción de la importancia de la agricultura en la seguridad alimentaria y el papel que ella juega en el camino hacia el desarrollo, Stern (2003, citado por A.D. Gillet, 2003) expone que "el desarrollo es fundamentalmente un proceso de cambio con procesos tan importantes como la creciente productividad e intensidad agrícola, el desplazamiento de personas de las fincas al sector industrial y de servicios y del campo a los pueblos y ciudades" y que asegurar la tenencia de la tierra, en especial para los pobres y las mujeres, cuyos derechos generalmente son ignorados, es una condición clave para este desarrollo, tal como lo es la capacidad de intercambiar derechos de propiedad a bajo costo.

Es evidente la necesidad de los gobiernos de disponer de arreglos sociales para hacer respetar los derechos de propiedad y de uso, sistemas eficientes y por lo mismo baratos de servicios para la transacción y obviamente servicios topográficos adecuados para evitar y resolver conflictos y con capacidad de constituirse en el sistema de soporte de la información física de las tierras para el catastro y el registro de títulos, un aporte decisivo también en el aseguramiento del capital de las familias 
rurales (Andrew, 2007). Un servicio óptimo debe ser capaz de ofrecer transparencia por la calidad de la información ofrecida y equidad en la posibilidad de acceso de los usuarios por su bajo costo.

El gran reto en Colombia es consolidar los derechos de propiedad sobre la tierra, que aunque abstractos, son una realidad jurídica y sociológica todavía en construcción, y hacer que ellos sean una institución social que promueve mejores niveles de vida y de convivencia, porque han sido dados dentro del marco de la legitimidad y son correspondientes con la realidad física en la medida que son soportados por un sistema que garantiza su demarcación para el respeto del límite de la propiedad.

Wallace (2009) ayuda a la comprensión de las actuales cuestiones de la tierra, cuando afirma que actualmente la caja de herramientas tiene elementos de la topografía geomática y del derecho, que universalmente han facilitado el derecho de acceder a las potencialidades del recurso suelo, para un nuevo constructo, que se anticipe a la controversia de la propiedad en si misma, por lo que buenos sistemas de información y demarcación acompañados de políticas públicas que favorecen el acceso a las potencialidades de la tierra, derechos de propiedad y derechos de uso, pueden hacer confluir los intereses públicos y privados en el camino hacia el desarrollo.

Al retomar el tema de los derechos de uso, es Tejo (2003) quien ahora aporta sus reflexiones acerca de la percepción de que el acceso a la propiedad ya no es el determinante principal en la capacidad de generar y apropiar riqueza como tampoco del cambio social, afirmándose en que es necesario reorientar la atención a la distribución equitativa de la educación, la capacitación y la información, y acometer la tarea de fortalecer las instituciones que faciliten el desarrollo de los mercados de derechos de uso de los factores, que requieren de condiciones jurídicas y económicas apropiadas, ello dice el autor, en igualdad con, o tal vez, mayor esfuerzo que el aplicado al desarrollo del mercado de los derechos de propiedad.

En Latinoamérica dejar de lado el esfuerzo por la redistribución de la propiedad y fomentar el arriendo de tierras como materialización de los derechos de uso es una tarea que tiene inherente un drástico cambio institucional, de hecho De Janvry, Macours y Sadoulet (2002) determinaron ocho limitantes al desarrollo de los mercados de arrendamientos: el temor de los terratenientes a perder las tierras que arriendan; miedo a que sean objeto de uso abusivo; arrendamientos limitados a círculo de personas conocidas por el terrateniente; arrendamientos socialmente segmentados; arrendamientos a corto plazo para evitar la posesión de facto de las tierras; restricciones legales sobre el arrendatario; prohibición del arriendo de tierras asignadas por medio de la reforma agraria, y las condiciones precarias del alquiler de terrenos pertenecientes a dueños ausentes.

La evolución hacia los mercados de arrendamientos sólo será posible si se acomoda el marco institucional que coadyuve en el aseguramiento de los derechos 
de uso: Derechos de propiedad claros, ambiente social seguro, arrendatarios con capacidad negociadora y administrativa y recursos básicos de financiación.

Los derechos de uso del suelo en el sentido amplio han sido tema de estudio académico y paulatinamente iniciativa de política pública, ellos son un cambio institucional que sin duda incidirán en otros cambios como la mayor fuerza decisiva que van toman las acciones locales, pues el arriendo simple o el arriendo con opción de compra, por ejemplo, como transacciones de tierras sólo se consolidan a partir de espacios donde hay confianza entre los agentes, por la novedad de la opción y mientras se conjugan aspectos del derecho consuetudinario con el derecho positivo que poco a poco se afianza.

Los autores hacen un llamado a considerar dentro de las iniciativas de política pública lo que UN-HABITAT (2008) ha encontrado: que los servicios formales de administración del suelo son más efectivos cuando se ofrecen a nivel local y que una opción es un establecimiento institucional local donde sólo sea necesario hacer una única solicitud para los servicios relacionados con la propiedad del suelo, lo mismo que la creación de concejos locales de tierras que representen y respondan a todos los intereses de las partes interesadas como las autoridades locales y consuetudinarias, propietarios de tierras, usuarios de suelo masculinos y femeninos y miembros de la comunidad.

Los logros institucionales para la administración de la tierra se obtienen más fácilmente a través de una gestión descentralizada, porque se puede responder a prioridades locales en vez de concentrar esfuerzos en satisfacer trámites burocráticos altamente centralizados, de igual modo sucederá con los sistemas de información territorial amplios y actualizados que puedan captar la complejidad de la ocupación actual, su uso y reivindicaciones, incluido el conjunto de derechos que se sobreponen, que es muy válido sobre todo en sociedades en situación postconflicto, como lo manifiesta Santfield (2006).

Los derechos de uso sobre la tierra conducirán a la consolidación de unas nueva ruralidad, en la que lo rural

"trasciende lo agropecuario, y mantiene nexos fuertes de intercambio con lo urbano, en la provisión no sólo de alimentos sino también de gran cantidad de bienes y servicios, entre los que vale la pena destacar la oferta y cuidado de recursos naturales, los espacios para el descanso, y los aportes al mantenimiento y desarrollo de la cultura" (Pérez, 2001, p.18).

Para la nueva ruralidad, la localidad, no necesariamente cabecera municipal, asume roles y responsabilidades del y para el desarrollo, y sus interrogantes están en la actualidad sobre las cuestiones del medio ambiente, el hábitat y los sistemas productivos. 
Localmente el territorio rural ocupado por actividades tradicionalmente agrarias ha estado colmado en los últimos tiempos por desarrollos de ingeniería, lineales como vías o tendidos eléctricos, o concentrados como hidroeléctricas, que estructuran nuevos paisajes y transforman flujos naturales, modifican ecosistemas y establecen nuevos patrones de ocupación y usos del suelo, globalmente, los avances en las ciencias y la tecnología han cambiado los patrones de consumo y por lo tanto aumentado y variado las demandas de productos naturales, estos cambios expresan la necesidad de nuevos acuerdos como los relacionados con el uso del suelo rural como productor en economías de escala, receptor de industrias o como sumidero de residuos, porque los nuevos usos indiscutiblemente ocasionaran externalidades sobre la propiedad rural.

El regreso a la producción limpia y la necesidad de las comunidades de aprovechar las ventajas comparativas, crearon una nueva noción de espacio territorial y la proximidad, ya no en función de la distancia sino en función de la densidad y la calidad de los sistemas de comunicaciones:

"Con toda evidencia, la más antigua división entre el campo y la ciudad se borra bajo nuestros ojos, y este hecho se revela como una de las más drásticas mutaciones que afectan a nuestra civilización. Se precipita la fusión entre el campo y la ciudad, (Dubby, 1995 citado por Zuluaga G.P, 2000)

Se necesita explorar para conocer con precisión el alcance logrado por las acciones orientadas a la optimización de los sistemas actuales de administración de la tierra, para no ser injustos en la evaluación de los esfuerzos destinados a esa tarea y se necesita la construcción de un estatuto rural que en el capítulo de tierras sea consecuente con la dinámica actual de uso y aplicaciones tecnológicas, este artículo espera ser un aporte para la iniciativa de políticas públicas encaminadas a que derechos de propiedad y derechos de uso se conjuguen hacia el logro del amento del nivel de vida en la población rural y por extensión de la población, en general.

\section{Referencias}

Andrew U. Frank (2007) Cadastre and Capital In GIM international Volume 21, Issue 9, The Netherlands ISSN 1566-907

Balcázar, Álvaro, López N., Orozco M. L. y Vega M. (2003) Colombia: Lecciones de reforma agraria En Mercados de tierras agrícolas en América Latina y el Caribe, Una realidad incompleta. Compilación de Pedro Tejo. CEPAL-GTZONU Santiago de Chile

Barnes, Grenville, (2002) Global Survey of Cadastral Experience, Lessons Learned: An Evaluation of Cadastral Initiatives En Latin America over the Past Two Decades FIG XXII International Congress TS7.3 Washington, D.C. USA 
Bejarano, J. Antonio (1998) Economía de la Agricultura. IICA Tercer Mundo Editores. Bogotá ISBN 958-601-811-3

(1999) Análisis económico del derecho: Cometarios sobre textos básicos. En Revista de Economía Institucional, No. 001, ISSN 0124-5996 Bogotá - Col. Pág. 155-167

\section{CONSTITUCIÓN POLÍTICA DE COLOMBIA 1991}

DANE (2009) Departamento Nacional de Estadística. Indicadores laborales y del sector agropecuario.[en línea] http://www.dane.gov.co/index.php?option=com frontpage \&Itemid=1 [citado en Marzo 18 de 2009]

Decreto 2811 del 18 de diciembre de 1974 CÓDIGO NACIONAL DE RECURSOS NATURALES RENOVABLES Y DE PROTECCIÓN AL MEDIO AMBIENTE DE COLOMBIA

Decreto Ley 1250 de 1970 Por el cual se expide el estatuto del registro de instrumentos públicos

De Janvry A. Sadoulet E. (2005) Access to Land and Development, University of California at Berkeley, Prepared as an entry for the New Palgrave Dictionary of Economics, 2nd edition, Palgrave Macmillan. [en línea] http://are.berkeley. edu/ sadoulet/papers/Palgrave.pdf [citado en Marzo 18 de 2009]

De Janvry, Macours y Sadoulet (2002) El acceso a tierras a través del arrendamiento En El acceso a la tierra en la agenda del desarrollo rural. Serie Informes técnicos. Departamento de desarrollo sostenible. BID 2002. RUR- 108

Deininger K. (2003) Land Policies for Growth and Poverty Reduction, World Bank. ISBN-13: 978-0-8213-5071-3

De la Garza T. Enrique (2005) Neoinstitucionalismo, ¿Opción ante la elección racional? Una discusión entre la Economía y la Sociología. En Revista Mexicana de Sociología Vol. 67, núm. 1 (enero-marzo, 2005): 163-203. México, D. F. ISSN: 0188-2503/05/06705-05

Demstez Harold (1967) Hacia una teoría de los derechos de propiedad, Traducido de American Economic Review, en recuperado en abril de 2009 del sitio web http:// www.eumed.net/cursecon/textos/Demsetz_teoria-derechos-propiedad.pdf

DNE DIRECCIÓN NACIONAL DE ESTUPEFACIENTES. Censo de cultivos ilícitos 2008. [en línea] http://www.dne.gov.co/?idcategoria=2574 [citado en abril 5 de 2009]

Banco de la República (1997) Urgentes medidas sobre tierras En DOCUMENTOS QUE HICIERON UN PAIS. Archivo Nacional de Colombia, biblioteca virtual [en línea] http://www.lablaa.org/blaavirtual/historia/docpais/indice.htm [citado en abril 5 de 2009] 
Echeverría Rubén, (1998) Un creciente interés en lograr mercados de tierras rurales más efectivos En Perspectivas sobre mercados de tierras rurales en América Latina. Informe técnico. Departamento de Desarrollo Sostenible. División de Medio Ambiente. Washington, D.C. Diciembre 1998 - No. ENV - 124

Gillet A.Davis, (2003) Los derechos de propiedad para los pobres como clave para la reducción de la pobreza y el crecimiento - Informe del Banco Mundial, nota periodística[en línea] recuperado de la página web del Banco Mundial, http:// web.worldbank.org/WBSITE/EXTERNAL/BANCOMUNDIAL/NEWSSP ANISH/0,,contentMDK:20116229 pagePK:64257043 piPK:437376 theSite PK:1074568,00.html [citado en abril 5 de 2009]

FAO (2003) Tesauro Plurilingue de Tierras, FAO, Organización de las Naciones Unidas para la Agricultura y La alimentación. ISBN 92-5-304283-4

Hayami, Yujiro and Vernon W. Ruttan. Agricultural Development. 2nd ed. Baltimore: Johns Hopkins University Press, 1985

Kalmanovitz Salomón. (2003) El neoinstitucionalismo como escuela. Publicación de Junta Directiva. Banco de la República. Bogotá, [en línea] e http://www.banrep. gov.co/junta/publicaciones/salomon/El_\%20neoinstitucionalismo_como_ escuela.pdf [citado en abril 5 de 2009

Ley 57 de 1857. CÓDIGO CIVIL COLOMBIANO

Ley 200 de 1936, SOBRE EL RÉGIMEN DE TIERRAS

Ley 135 de 1961, SOBRE REFORMA SOCIAL AGRARIA

Ley 30 de 1988, POR LA CUAL SE MODIFICAN Y ADICIONAN LAS LEYES 135 DE 1961, 1a. DE 1968 Y 4a DE 1973 Y SE OTORGAN UNAS FACULTADES AL PRESIDENTE DE LA REPÚBLICA

Ley 99 de 1993. LEY DEL MEDIO AMBIENTE COLOMBIANO

Ley 160 de 1994, POR LA CUAL SE CREA EL SISTEMA NACIONAL DE REFORMA AGRARIA Y DESARROLLO RURAL CAMPESINO

Ley 1152 de 2007, ESTATUTO RURAL COLOMBIANO

Machado Absalón y Torres Jorge. (1991) El Sistema Agroalimentario. Coedición de CEGA y Siglo XXI editores. Bogotá ISBN958-606-008-X

(2002) De la Reforma Agraria a la Reforma Rural En Colombia Tierra y Paz. Instituto Colombiano de la Reforma Agraria INCORA. Bogota. ISBN 95833-3004-3

Martínez, Yovanny y Ubaque, Nyrian Angélica. (2002) Regional Experience in the Cadastre - Africa and the America.s. Management and Modernization of the Cadastral Infrastructure in Colombia TS7.1 FIG XXII International Congress Washington, D.C. USA 
Mckenna Jack and Urban-Karr Jill (2008) Secure Land Tenure, Land rights for women and the poor en GIM INTERNATIONAL. Volume 22, Issue 12. The Neerlands,ISSNN 1566-9076

Molina J. (2009) Seguridad Alimentaria y Reforma Agraria en documento recuperado el 12 de mayo de 2009 del sitio web de la FAO: http://www.rlc.fao. org/es/desarrollo/interag/pdf/segualim.pdf

North Douglas. (1995) Instituciones, cambio institucional y desempeño económico. Fondo de cultura económica, economía contemporánea. Reimpresión México 1995. ISBN 9681639820

Pérez E. y Llambí LuIs. (2007) NUEVAS RURALIDADES Y VIEJOS CAMPESINISMOS. Agenda para una nueva sociología rural latinoamericana. En Cuadernos de Desarrollo Rural, Vol. 4 No. 59. Pág. 37-61. Pontificia Universidad Javeriana. Bogotá ISSN 0122-1450

Pérez E. (2001) ¿Hacia una nueva visión de lo rural En Una nueva ruralidad en América Latina? Compilación de Norma Giarracca, CLACSO, Buenos Aires. ISBN 950-9231-58-4

Rivas Leone José Antonio (2003) El neoinstitucionalismo y la revalorización de las instituciones. En Reflexión Política, año 5 No 9 IEP - UNAB Bucaramanga. Colombia ISSN 0124-0781

Rojas Manuel (2006) Políticas de tierras, reforma agraria y reconstrucción del territorio rural en Colombia, En Reforma Agraria y desarrollo rural en la Región Andina. CEPES, Editor Fernando Eguren. Lima. Perú

Santa Biblia, Libro de Josué, Capítulo 18, verso 8. Versión Reyna Valera. 1960

Santos L. E y Lozano R. (2008) Factores que inciden en el precio de las tierras de uso agrícola en la provincia de Mares, Departamento de Santander. En Revista CIFE No. 13 Universidad Santo Tomás, Bogotá. Cife ISSN: 0124-3551

Stanfield, Safar, Leka y Manoku. (2006) Reconstruction of Land Administration in Post Conflict Conditions, [on line] Symposium on Innovative Technology for Land Administration

http://www.terrainstitute.org/pdf/rcon_Landadmin\%20PstCnflct\%20.pdf [documented in august 15 2009]

Tejo, Pedro et al. (2003) Mercados de tierras agrícolas en América Latina y el Caribe: una realidad incompleta. Publicación de Naciones Unidas y CEPAL. Santiago de Chile, Chile 2003 ISBN 92-1-322207-6

Ting, Lisa y Williamson Ian.(2009) Land Administration and Cadastral Trends: The Impact of the Changing Humankind-Land Relationship and Major Global Drivers en The University of Melbourne. [on line] 
http://repository.unimelb.edu.au/10187/1289 [documented in june 18 2009]

UN-HABITAT (2008) DERECHOS SEGUROS AL SUELO PARA TODOS, United Nations Human Settlements Programme (UN-HABITAT), Nairobi Kenya, ISBN:978-92-1-132015-2

Usaquén Chía Martha I. (2008) Externalidades: Más que un problema de derechos de propiedad En Revista CIFE Universidad Santo Tomás, Año 10 No. 13. ISSN 0124-3551 Bogotá

Valdivieso S. (2001) North y el cambio histórico: Luces y sombras de la nueva historia institucional En Revista de Economía Institucional No. 4. 157-152

Vogelgesang Frank (2003) Derechos de propiedad, costos de transacción, externalidades y mercados de tierras rurales en América Latina y el Caribe, en: Mercados de tierras agrícolas en América Latina y el Caribe: una realidad incompleta, compilación de Pedro Tejo. Publicación de las Naciones Unidas ISBN: 92-1-322207-6 LC/G.2202-P. Impreso en Naciones Unidas, Santiago de Chile Julio de 2003

Wallace Judy (2009) Making Land Markets Work for All, This paper was prepared and presented for the first time in session MKTl: Potential and Challenges for Land Markets at the FIG and World Bank Conference on Land Governance in Support of the MDGs: Responding to New Challenges, Washington DC, USA, 9-10 March 2009

Williamson Ian (2008) Using Cadastres to Support Sustainable Development, In Spanish IX National Congress of Surveying Engineers TOP-CART 2008 in Valencia, Spain 18-21 February 2008 [on line]

http://www.fig.net/pub/monthly_articles/april_2008/april_2008_williamson. html [documented in june 18 200]

Zuluaga Gloria P. Las nuevas funciones del espacio rural. Artículo publicado en Ensayos Frohum [En línea] 15 (2000)

http://agora.unalmed.edu.co/docs/For15-5-Zuluaga.PDF [citado en julio de 2009] 